\title{
Erratum: A statin-loaded reconstituted high-density lipoprotein nanoparticle inhibits atherosclerotic plaque inflammation
}

Raphaël Duivenvoorden, Jun Tang, David P. Cormode, Aneta J. Mieszawska, David Izquierdo-Garcia, Canturk Ozcan, Maarten J. Otten, Neeha Zaidi, Mark E. Lobatto, Sarian M. van Rijs, Bram Priem, Emma L. Kuan, Catherine Martel, Bernd Hewing, Hendrik Sager, Matthias Nahrendorf, Gwendalyn J. Randolph, Erik S.G. Stroes, Valentin Fuster, Edward A. Fisher, Zahi A. Fayad \& Willem J.M. Mulder

Nature Communications 5:3065 doi:10.1038/ncomms4065 (2014); Published 20 Jan 2014; Updated 19 Mar 2014

The affiliation details for Edward A. Fisher are incorrect in this Article. The correct affiliation details for this author are given below: Department of Medicine (Cardiology) and Cell Biology, Marc and Ruti Bell Program in Vascular Biology, NYU School of Medicine, New York, New York 10016, USA. 